\title{
Impressioni storiche: Parma dal fascismo alla Liberazione nelle foto dell'Archivio Amoretti
}

Alla Casa della musica di Parma, dal 25 aprile al 24 maggio 2015, una mostra fotografica promossa dall'Assessorato alla cultura, con la consulenza scientifica dell'Istituto storico della Resistenza e dell'età contemporanea di Parma, ha esposto 150 scatti di Armando Amoretti. In questo articolo gli autori presentano la storia dello studio fotografico Amoretti e una selezione di immagini tratte dallo straordinario fondo archivistico lasciato dal fotografo parmense.

At the House of Music of Parma, from April 25 th to May 25 2015 , the photographic exhibition organized by the Municipality of Parma (Assessorato alla cultura) with the scientific advice of the Historical Institute of the Resistance and the Contemporary Age of Parma, exhibited 150 photos of Armando Amoretti. In this paper the authors present the history of the Amoretti's atelier and a selection of his extraordinary photographic archive.

\section{Studio fotografico Amoretti, di Andrea Tinterri}

Ventiquattro fotografie per un evento. Un racconto che circoscrive un luogo e una durata: lo spazio della strada e il momento dell'attesa. Sono queste le due coordinate che trasformano gli scatti di Armando Amoretti, realizzati nell'agosto del 1922 a Parma durante le barricate, in immagini esemplari, icone, in qualcosa di sintetico capace d'esprimere i limiti dello spazio e il sentimento della durata. All'epoca Armando Amoretti lavorava presso lo studio del fotografo Vaghi, ma alle spalle aveva già quasi sette anni di apprendistato. Terminata la terza elemen- 


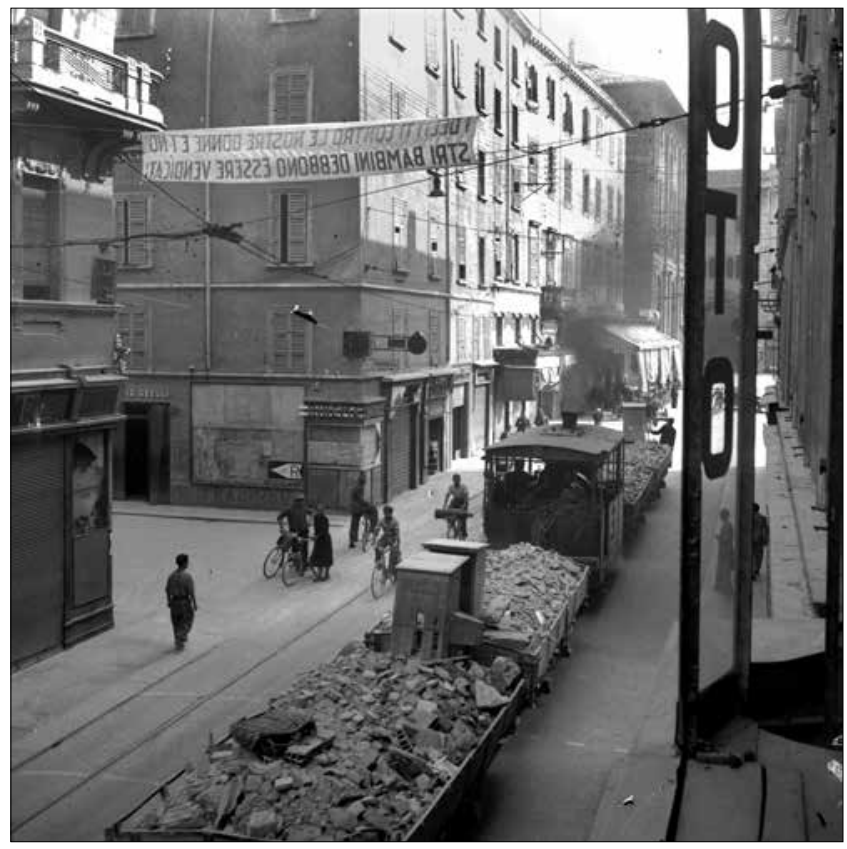

Dallo studio Amoretti, via Cavour, 1944

tare iniziò a lavorare presso un negozio di macchine da cucire, un breve periodo che anticipò l'accesso alla società fotografica e alle diverse migrazioni a cui sarebbe stato costretto.

All'età di tredici anni affiancò il fotografo Ettore Pesci, un impegno attraverso cui iniziò a riflettere sulle azioni della fotografia: ripresa, stampa, ritocco. Un lavoro di "bottega" che aveva i suoi segreti da custodire. Come ricorda Giovanni Amoretti, in alcune occasioni Ettore Pesci invitava Armando a uscire dallo studio e aspettare sul pianerottolo, per evitare che l'apprendista acquisisse i preziosi "trucchi del mestiere", le chiavi d'accesso alle abilità conquistate sul campo.

È interessante riflettere sull'eterogeneità delle funzioni svolte, dalla stampa, al ritocco passando per quello che oggi definiremmo il "lavoro di assistente". Questo aiuta a contestualizzare il mestiere del fotografo nel primo decennio del Novecento. Un lavoro complesso ma unitario: scatto, stampa, postproduzione. Un percorso organico in cui nessuna competenza veniva delegata ad un operatore esterno allo studio. La parola "onestà" sembra riportarci a quel ricordo, a quel racconto "del pianerottolo", al fatto che tutto doveva rimanere dentro le mura in cui si lavorava. Onesti verso i segreti, un'omertà richiesta e necessariamente restituita.

Armando abbandonò il mezzo fotografico per un incarico in fureria. Le informazioni raccolte dal figlio Giovanni non sono precise sul periodo tra il $1916 \mathrm{e}$ il 1918. Il dato certo è la presenza di Armando in Albania, dove si ammalò di 
malaria, ma rimane piuttosto vaga la durata della sua permanenza nel territorio balcanico. Terminata la guerra, intenzione di Armando era chiedere il congedo a causa della malattia riscontrata ma, su consiglio del suo medico curante, accettò il trasferimento in Libia. Il clima secco avrebbe aiutato il decorso della malattia $\mathrm{e}$, finalmente, gli furono riconosciute le sue capacità lavorative. Fu inviato quindi come fotografo militare, ma del materiale realizzato rimangono pochissime tracce. Nell'archivio Amoretti è possibile rintracciare una sequenza di scatti che descrivono una panoramica completa del porto libico e alcune fotografie personali che vedono Armando in divisa.

Tornato in Italia, lavorò qualche mese a fianco del fotografo Pisseri, ma questa rappresentò solo una breve parentesi prima di entrare a far parte dello studio Vaghi, un luogo in cui la fotografia era anche politica, era anche il racconto ufficiale e austero dei ritratti di Casa Savoia, di Benito Mussolini seduto dietro ad una scrivania, dei gerarchi del partito, Galeazzo Ciano, Italo Balbo, Giuseppe Bottai, per citarne solo alcuni.

Nel 1922 Luigi Vaghi diventò il fotografo ufficiale della Casa Reale, lo stesso anno in cui inizia il nostro racconto, quello di Armando Amoretti. E non è un caso che le date coincidano: una sovrapposizione temporale che ci permette di capire il motivo per cui fu il giovane apprendista a essere inviato nell'Oltretorrente parmense a documentare un momento di resistenza, il momento prima dell'incubo. Luigi Vaghi non si poteva esporsi in prima persona nei borghi della città socialista, non poteva immischiarsi nelle vicende di un lotta popolare che tentava di respingere quell'Italo Balbo che poco dopo avrebbe fotografato. Ma non poteva nemmeno perdersi l'evento, il classico appuntamento con la Storia, e quindi incaricò Armando, che quei borghi li abitava, li frequentava, e con i quali aveva in comune la stessa tradizione politica.

Ed è in questo modo che nasce il racconto delle barricate di Parma del 1922, ventiquattro scatti che narrano un'attesa nel possibile luogo dello scontro: la strada. Amoretti si trovò di fronte a un problema, come fotografare costruzioni occasionali che sarebbero servite come protezione: barriera popolare a un agente esterno. Al momento degli scatti l'incursione era qualcosa di possibile, ma ancora distante nel tempo. C'erano solo muri, barricate, fili spinati, non c'era battaglia, non c'era l'invasore: solo un elemento a ricodificare la linearità dei borghi, a ridefinire l'occupazione delle vie di comunicazione.

Erano due gli aspetti da cogliere in quelle ore. La struttura in quanto tale, quindi la "materia" della nuova città, e la strada restituita nella sua estensione massima, dall'alto verso il basso, dai piani superiori delle case fino a raggiungere gli ultimi sassi del selciato. Perché le barricate sono qualcosa di cui va analizzata 
la successione. Tra una barriera e l'altra si creano spazi chiusi, entro cui abitare, entro cui raggrupparsi e aspettare. La fotografia scattata dall'alto - Armando salì su un piano alto della casa e inquadrò borgo dei Minelli - risulta essere l'esempio perfetto di quella che potremmo definire l'iconografia della barricata. Infatti il fotografo delimita l'immagine attraverso due edifici, gli stessi che aprono la via, due strutture da cui inizia il percorso dello sguardo che accompagna l'intera estensione della strada, fino alla chiesa del quartiere che chiude il teatro dell'evento. Il fotografo si trovava all'interno di un luogo privato, un'abitazione di cui probabilmente conosceva il proprietario, e guardava fuori, osservava il territorio che sarebbe diventato il campo della collisione tra due entità diverse, politicamente e culturalmente differenti. E poteva essere solo lo spazio pubblico il luogo dell'incontro, del duello. Armando sembra implicitamente percepirlo restituendo una visuale ampia e non circoscritta al singolo oggetto. L'inquadratura racconta la struttura urbana senza censurare i tetti delle case, i cornicioni, come se fosse necessario restituire l'intero spazio del possibile scontro e la successione delle nuove costruzioni. Sembra quasi una forma di rispetto nei confronti dei borghi della città, dello spazio pubblico, del luogo dello scambio. Il modo di inquadrare ci permette anche di capire come queste barriere estemporanee modificassero la partecipazione alla strada stessa. Piccoli gruppi di persone a creare un'immaginaria serpentina lasciando libero un corridoio centrale, come se dessero al fotografo la possibilità di cogliere l'intera estensione dello spazio pubblico e l'idea di attesa: un tempo intermedio tra un prima (la costruzione) e un dopo (la resistenza). Un'inquadratura che riveli la trasformazione architettonica e sociale dell'insediamento urbano. Ma anche quando il punto di vista si abbassa e l'attenzione ricade sulla struttura della barricata (osserviamo ad esempio la fotografia che ritrae via della Salute) rimane evidente l'attenzione al contesto circostante, al quartiere, al luogo della resistenza. Armando si posiziona all'inizio della via, esattamente di fronte alla barriera difensiva. La ripresa è centrale, simmetrica, in maniera da evidenziare l'incastro artigianale a comporre la barricata, ma contemporaneamente viene evidenziata l'intera fisionomia dello spazio/strada. La città non è censurata, anzi le inquadrature di Armando conferiscono una sorta di dignità allo spazio pubblico ferito, necessariamente modificato per resistere a un'aggressione esterna, a un cancro. Mi vengono in mente le fotografie della città di Beirut (1991) di Gabriele Basilico. Un luogo massacrato dalla guerra che, però, conservava una sua compostezza architettonica che il fotografo milanese ha voluto rispettare, immortalando la città come se essa non avesse subito nessuna violenza, come se le armi non l'avessero invasa, come se non avesse subito nessuno stupro di massa. La collaborazione con lo studio Vaghi proseguì fino al 1938 e Armando andò 
specializzandosi nel ritocco fotografico. Un'abitudine professionale che segnò anche fisicamente gli strumenti del lavoro: le mani. Ricorda Giovanni Amoretti che il padre Armando aveva le punte delle dita segnate dalle schegge di grafite che lasciavano un pigmento scuro, quasi un segno di riconoscimento, un'eredità del mestiere.

Il grado di specializzazione gli consentì di avere un'alta considerazione all'interno dello studio Vaghi al punto da ottenere la custodia delle chiavi dei cassetti che contenevano le lastre che ritraevano Mussolini e i componenti della Casa Reale. Diritto questo di cui godeva, oltre allo stesso Armando, solamente Bruno Vaghi, figlio del fondatore Luigi. E fu proprio una fotografia del re Vittorio Emanuele III a definire la rottura con lo studio e la decisione di aprirne uno proprio in via Walter Branchi (attuale via Cavestro). In una fotografia realizzata da Luigi Vaghi, un particolare ne falsificava l'immagine: gli occhi chiari del re apparivano scuri. Una delle prime fotografie con negativo pancromatico e un problema da risolvere, una postproduzione necessaria perché lo scatto potesse essere accettato. Si incaricò Armando del ritocco, un lavoro che avrebbe restituito credibilità alla fotografia. È sempre Giovanni a ricordare il racconto del padre: Luigi promise ad Armando una ricompensa generosa nel caso fosse riuscito nel lavoro perché questo avrebbe consentito allo studio di vendere un quantitativo di stampe assai elevato, come in effetti successe. Ma come ringraziamento per la capacità dimostrata, gli fu offerta solo una cena. Fu quello l'episodio che gli fece capire che mai sarebbe avanzato di ruolo. Era il 1938 quando, con l'aiuto dei nonni materni, Armando aprì lo studio fotografico Amoretti.

\section{La città palcoscenico di storia, di Raffaella Ilari}

La fotografia è atto di memoria, per eccellenza. Chissà se un fotografo è sempre consapevole della responsabilità che ha nel preciso momento in cui il suo occhio ferma un'immagine in uno scatto e quell'immagine si fa subito testimonianza, travalica il concetto di temporalità e diventa patrimonio di memoria storica.

Le immagini eroiche delle barricate di Parma, della moltitudine festante per la visita del Duce, della sfilata partigiana nel primo anniversario della Liberazione, della folla oceanica del comizio di Palmiro Togliatti del 1948 in piazza Garibaldi, arrivano a noi attraverso lo sguardo del fotografo Giovanni Amoretti.

Immagini di altissima teatralità, messe-in-scene narrative di cui ancora oggi respiriamo la polvere, il silenzio, l'attesa, il caldo, in cui percepiamo, dietro l'enfasi retorica di un'iconografia fascista ormai prossima al crollo, paura e sofferenza. Noi spettatori non vediamo tutto, solo una parte. In questo gioco teatrale di scene 
e controscene assistiamo ad esempio alla celebrazione del regime ma non alla sofferenza e al dissenso politico per nulla assenti.

Un archivio visivo di memoria straordinario è questo che Amoretti ci ha lasciato, come si può osservare dalle immagini qui racchiuse, una preziosa documentazione sulla città come spazio pubblico in cui la storia viene partecipata, combattuta, messa in scena, celebrata, inneggiata, liberata. Strade, muri, barricate, fili spinati, i borghi, quei borghi che Amoretti antifascista conosceva assai bene: la città diventa palcoscenico di storia a partire dal luogo della piazza, gran teatro di rappresentazione.

In questo suo guardare il paesaggio, tra immagini private e di solitudine e altre di partecipazione pubblica popolare, tra luoghi deserti e altri all'opposto incredibilmente saturi, tra il silenzio dell'attesa e il rumore festante delle parate, Amoretti ha saputo in modo sublime raccontare la relazione tra le persone e i luoghi così come la trasformazione di un popolo agli eventi della storia.

La mostra è suddivisa per scene. Nella prima abbiamo le immagini di quel 28 ottobre 1939 in cui a Parma, come in tante altre città italiane, viene celebrato l'anniversario della marcia su Roma con un corteo che sfila per le vie del centro storico sino al cortile della Pilotta dove avvengono le orazioni ufficiali. Ma il
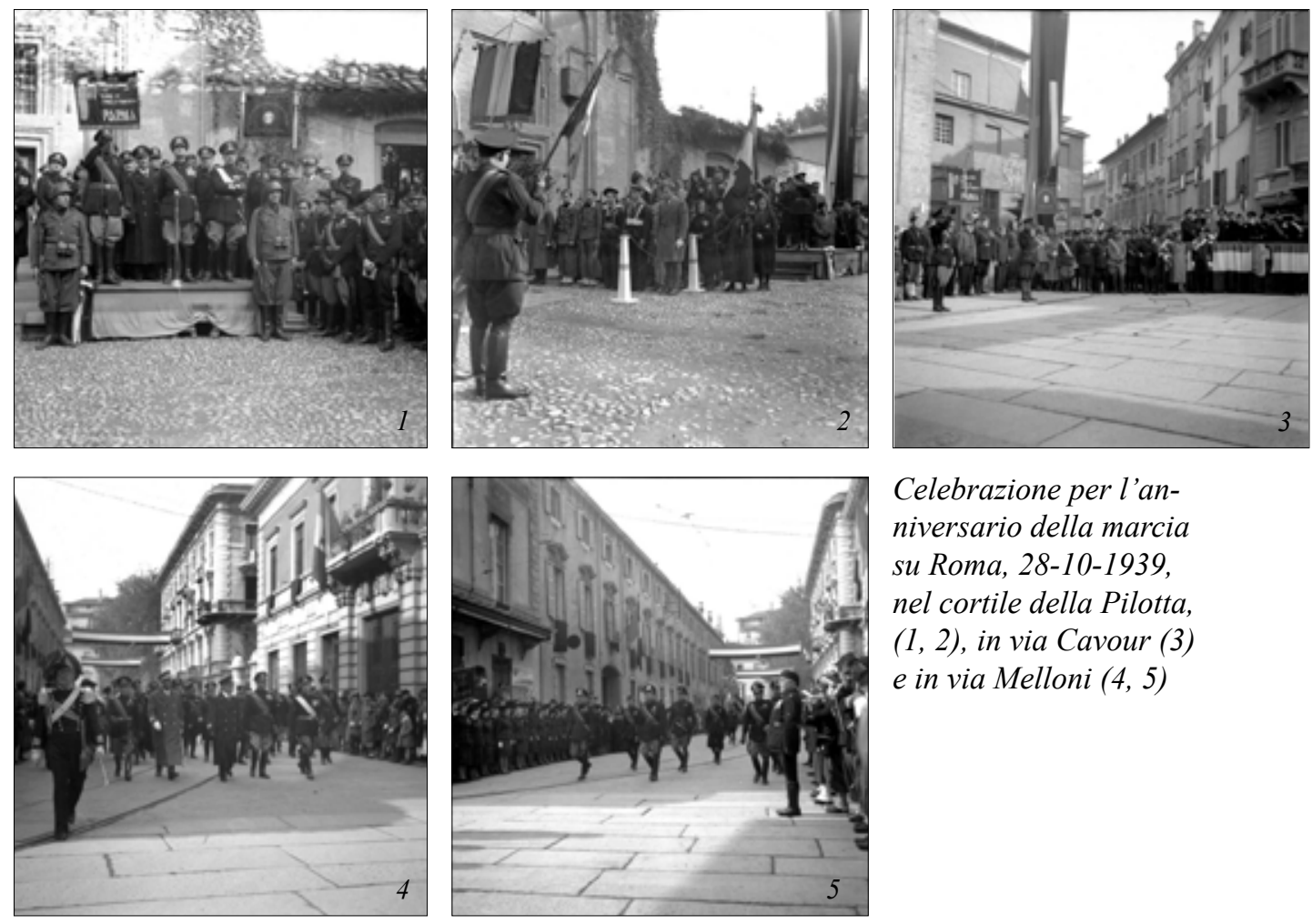

Celebrazione per l'anniversario della marcia su Roma, 28-10-1939, nel cortile della Pilotta, $(1,2)$, in via Cavour (3) e in via Melloni $(4,5)$ 
1939 è anche il ritorno dei legionari dalla guerra civile spagnola: di questo la mostra presenta fotografie di parate che dalla stazione si muovono prima verso via Toschi per la deposizione di corone al monumento della Vittoria, poi alla lapide in ricordo dei caduti del fascismo parmense in via Melloni e in piazzale Corridoni sino a via Bixio e a piazzale Rondani.

Il secondo blocco di foto è dedicato alla visita ufficiale di Mussolini avvenuta l's ottobre 1941 tra Parma, Fidenza e Salsomaggiore per consegnare la Spiga d'oro dell'anno XVIII dell'era fascista ai rurali parmensi, per il loro lavoro di sostegno nella "battaglia del grano". Vediamo il Duce visitare prima la Scuola militare di applicazione e poi piazza Garibaldi, atteso da una folla festante, e attraversare, a bordo della decappottabile, alcune vie del centro storico, addobbate con striscioni tricolori o neri, salutato ai lati della strada da donne, giovani Balilla, dopolavoristi. In campagna il premio della Spiga d'oro viene consegnato a contadini e tecnici in un'atmosfera di festa tra fisarmoniche e canti popolari. In Oltretorrente le foto ritraggono il Duce prima in piazzale Corridoni, poi in piazzale Picelli, sino ad arrivare al parco Ducale. Il popolo all'occhio di Amoretti sembra stringersi
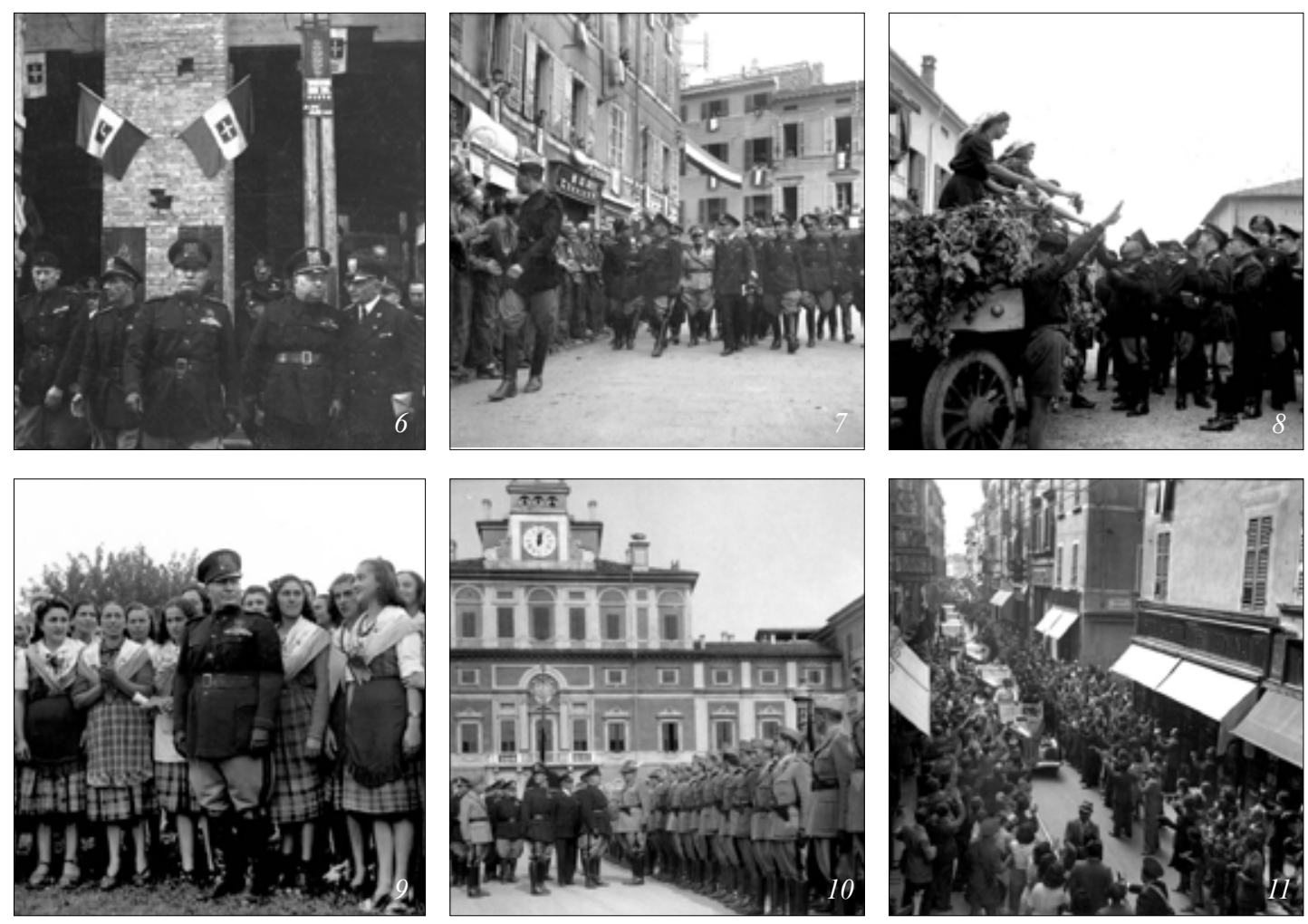

Visita di Mussolini a Parma, 08-10-1941 (6, 7), nella campagna parmense (8, 9), alla Scuola Militare di Applicazione (10) e in via Mazzini (11) 

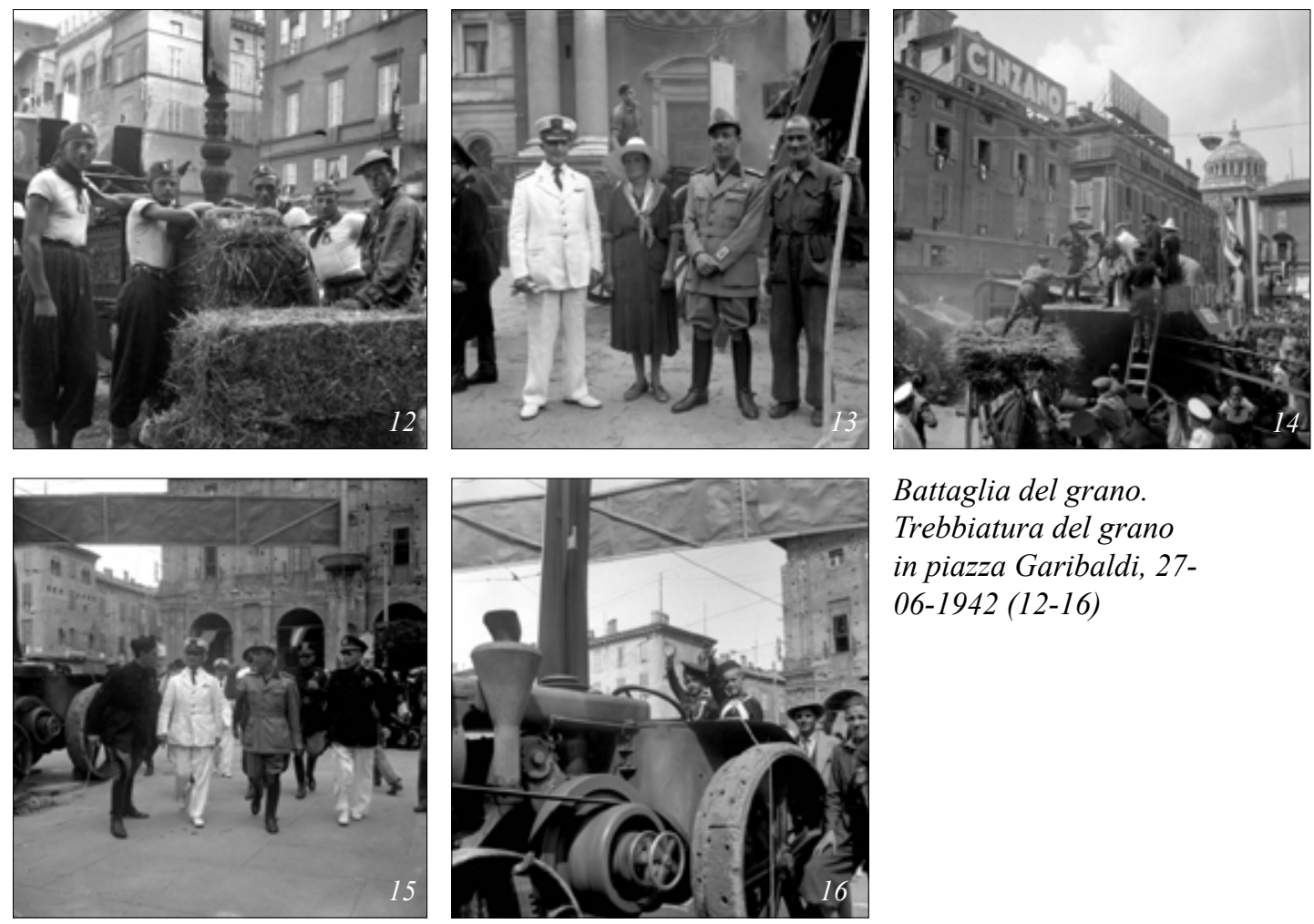

Battaglia del grano.

Trebbiatura del grano

in piazza Garibaldi, 27-

06-1942 (12-16)

convinto attorno a lui, ma è solo apparenza in quanto paura, angoscia e dissenso politico al regime non sono per nulla assenti.

Se la scena terza mostra la trebbiatura del grano del 27 giugno 1942, grande mobilitazione di massa fascista di propaganda, evento demagogico e nazionalista, in una piazza Garibaldi piena di trebbiatrici, grano e macchine agricole, la scena quarta raccoglie le immagini della celebrazione dei ventennali dei Fasci avvenuta nel cortile del palazzo della Pilotta. Tutto è solenne, ostentato e anche funereo dietro l'apparato di una rappresentazione prossima al tracollo.

La scena quinta narra la visita nel 1943 di Umberto di Savoia alla Scuola di applicazione di fanteria a palazzo Ducale, visita finalizzata a mostrare la vicinanza tra Casa Savoia e il regime fascista. Vicinanza che stava per concludersi con l'arresto di Mussolini su ordine del re, la firma dell'armistizio dell' Italia con gli alleati e il dissolvimento dello stato fascista lasciando macerie e distruzione.

La scena sesta ritrae Parma dal 1945 al 1948. Ormai disarmati, i partigiani sfilano per le vie del centro. I volti sono sorridenti. In uno scenario di macerie ancora visibili sui palazzi lacerati dai bombardamenti, nell'aria si respira gioia, serenità, orgoglio, fierezza. Lo stato totalitario è alle spalle, nasce la democrazia repubblicana. Piazza Garibaldi torna a vivere e a riempirsi in occasione dei comizi elet- 

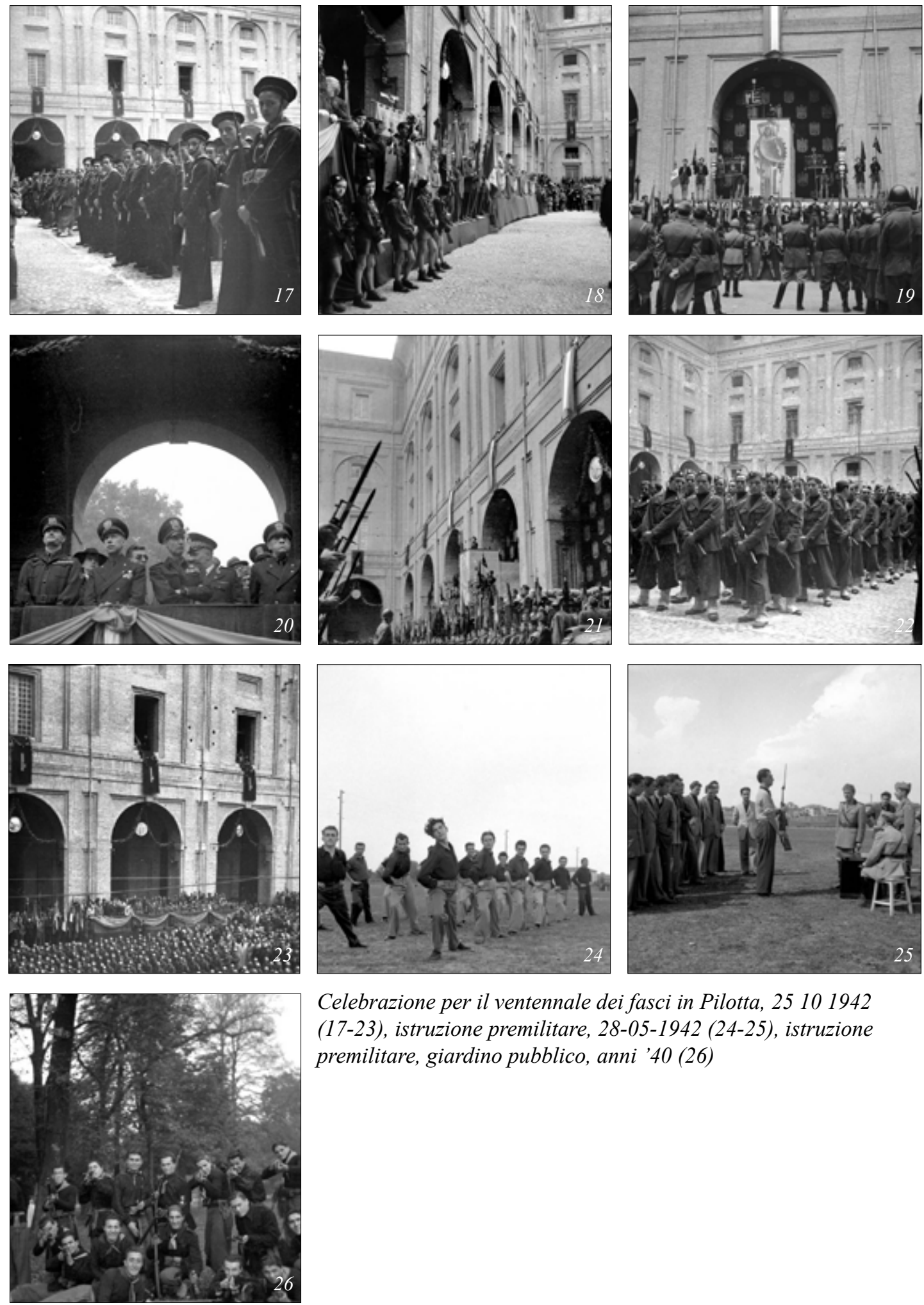

Celebrazione per il ventennale dei fasci in Pilotta, 25101942 (17-23), istruzione premilitare, 28-05-1942 (24-25), istruzione premilitare, giardino pubblico, anni '40 (26) 

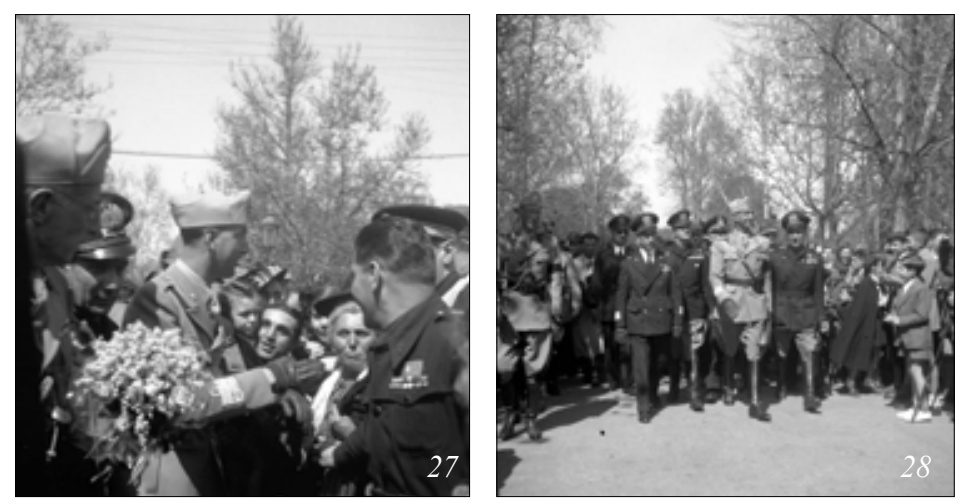

Visita di Umberto di Savoia alla Scuola di Applicazione, 18-04 $1943(27,28)$
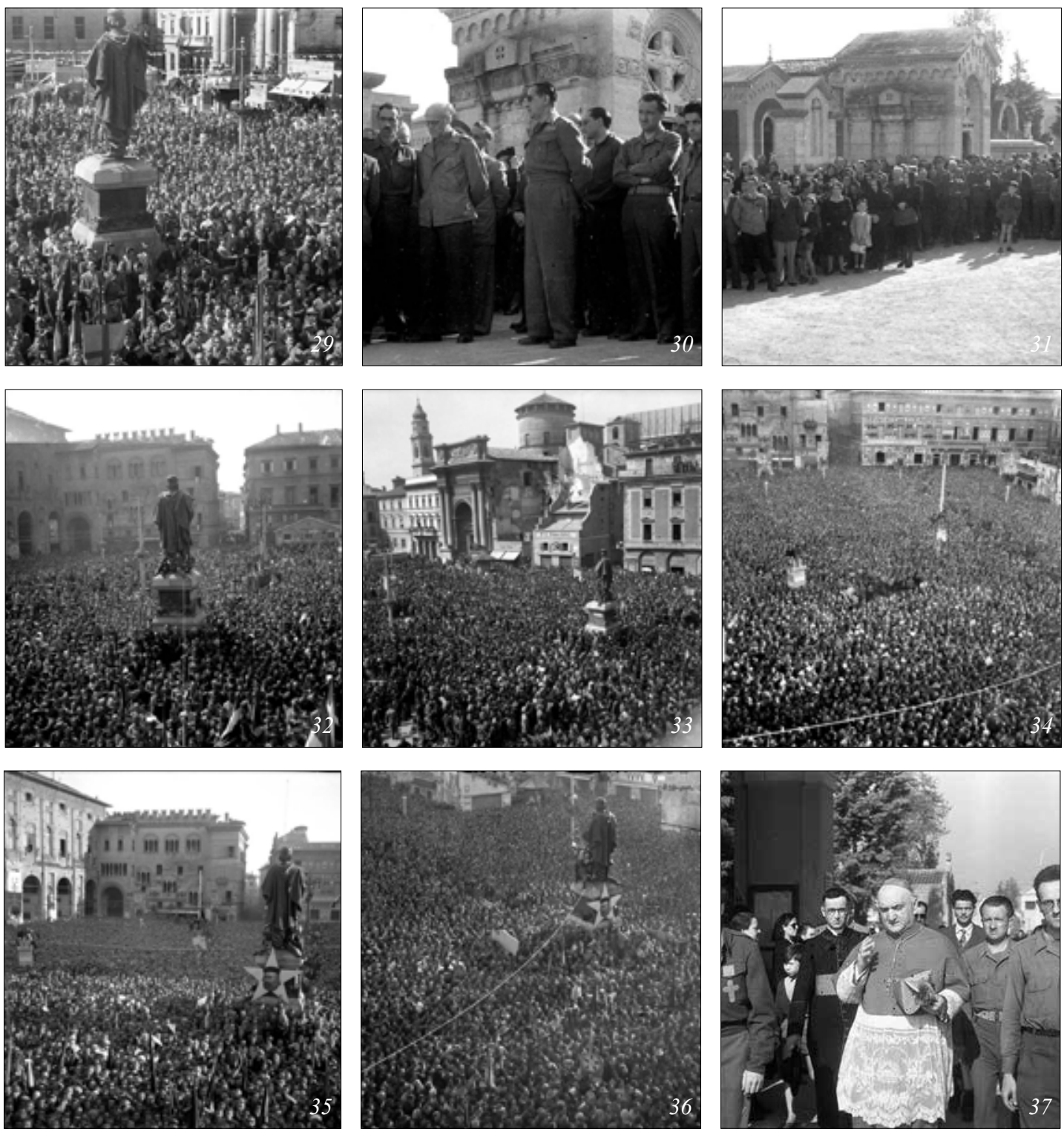

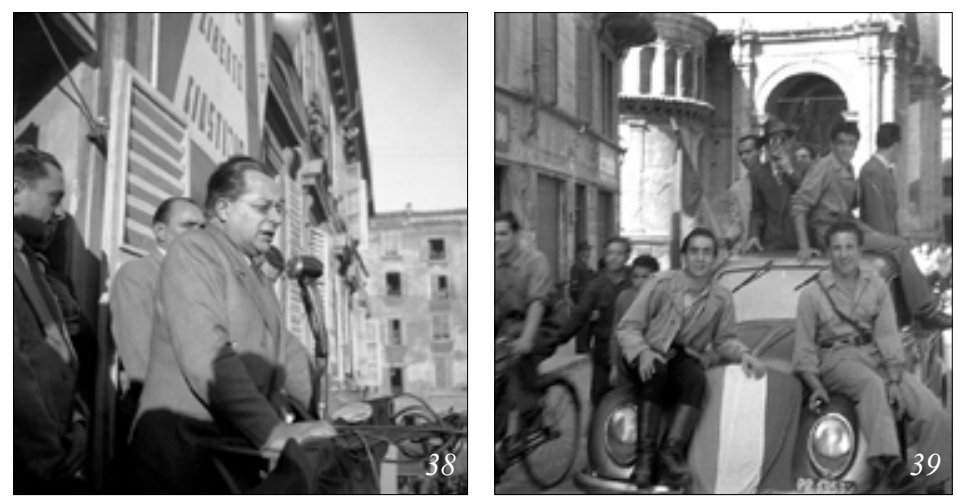

Arrivo della sfilata dei partigiani in piazza Garibaldi, 09-05-1945 (29), celebrazione in onore dei caduti per la Liberazione, cimitero della Villetta, 09-05-1945 (30, 31), celebrazione primo anniversario della Liberazione, piazza Garibaldi, 25-04-1946 (32, 33), comizio per la campagna elettorale, piazza Garibaldi, 12-04-1948 (34-36), l'arcivescovo Evasio Colli insieme ai preti partigiani (37), Palmiro Togliatti dal palazzo del Governatore durante il comizio per la campagna elettorale, piazza Garibaldi, 12-04-1948 (38), partigiani in via Vittorio Emanuele (attuale via della Repubblica) 09-05-1945 (39)

torali come testimonia la fotografia della folla oceanica del 12 aprile 1948 per la presenza di Palmiro Togliatti, segretario del Partito comunista italiano.

25 aprile 1945. La memoria è liberata. E liberandosi la memoria si inizia a raccontare, a testimoniare, attraverso racconti e immagini, di quel popolo che si era opposto alla dittatura fascista. Documento straordinario dell'Archivio Amoretti sono le 24 immagini delle barricate nell'estate del 1922, che documentano la prima resistenza antifascista di Parma e che ora trovavano un forte legame con la Resistenza da poco conclusa.
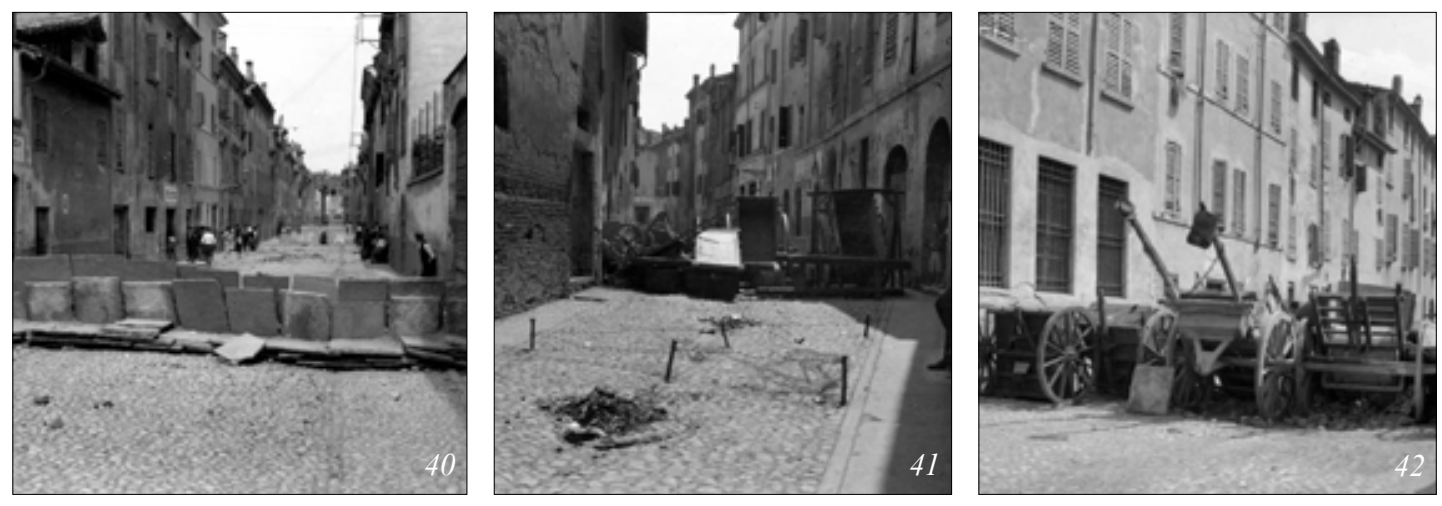

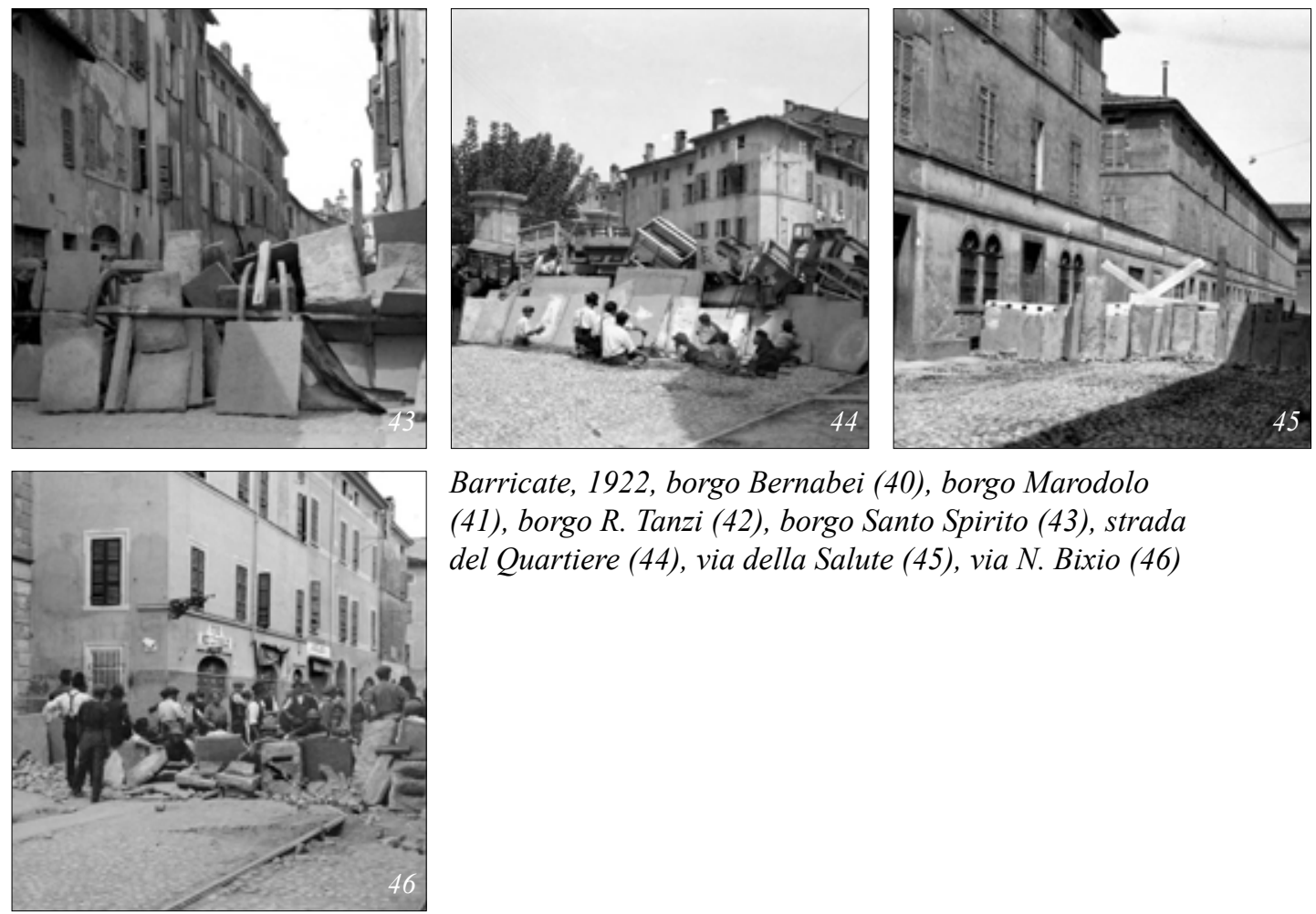

Barricate, 1922, borgo Bernabei (40), borgo Marodolo (41), borgo R. Tanzi (42), borgo Santo Spirito (43), strada del Quartiere (44), via della Salute (45), via N. Bixio (46) 